\title{
Differential gene expression in Lavandula angustifolia Mill. under adaptation ex vitro
}

Tsyupka V.A.*, Kuleshova O.N., Emirsaliev A.O., Zhdanova I.V., Mitrofanova I.V. Federal State Funded Institution of Science "The Labor Red Banner Order Nikita Botanical Gardens National Scientific Center of the RAS", Nikita, Yalta, Russia

*email:valentina.brailko@yandex.ru

Propagation of commercial lavender (Lavandula angustifolia Mill.) cultivars in vitro has a number of advantages over seed or vegetative (cuttings) propagation: obtaining a uniform, genetically stable and healthy planting material. At the same time, the effectiveness of this process depends on the subsequent adaptation of the obtained plants to the open-field conditions. The research was carried out on the 'Prima' cultivar in the collection of the Nikita Botanical Gardens. Leaves were collected from plants grown in vitro before transferring them to in vivo conditions and from plants after 7 days adaptation in a Conviron multilevel plant growth chamber (Canada). Lavender plants were cultured in vitro on MS culture medium supplemented with $0.5 \mathrm{mg} / \mathrm{L} \mathrm{BAP}$ or $0.5 \mathrm{mg} / \mathrm{L}$ kinetin and $0.025 \mathrm{mg} / \mathrm{L} \mathrm{NAA}$ at a temperature of $22-25{ }^{\circ} \mathrm{C}, 14-16$-hour photoperiod, light intensity $-25.0-37.5 \mu \mathrm{mol} \mathrm{m} \mathrm{m}^{-2} \mathrm{~s}^{-1}$. To induct rhizogenesis (after third passage). MS medium with different content of auxins, IBA or NAA $(0.5-1.0 \mathrm{mg} / \mathrm{L})$, was used. To analyze possible molecular mechanisms, which are the base of the adaptive processes in plants, at the time of transition from in vitro growth to in vivo growth the sampels of total RNA were isolated from leaves by the NucleoSpin RNA Plant protocol (NucleoSpin, Germany) and 24 barcoded RNA-Seq libraries were created using Illumna TruSeq Stranded mRNA Library Prep Kit. Sequencing was made on a high-performance sequencer Illumina NextSeq 550 using the NextSeq 500 HighOutputv2 Kit with an estimated capacity of at least 90 million reads. As a result of sequencing, 3 libraries of transcriptomic data were obtained for each of the states (in vitro and in vivo). For the obtained data, pre-preparation, de novo transcriptome assembly, transcript-level expression analysis, search for protein-coding regions and their annotation using the SwissProt Viridiplantae database, and evaluation of differential expression were made. The transcripts of up-regulation and down-regulation have been identified. The following software packages were used: FastP v. 0.19.5, Trinityv.2.11.0, Kallistov.0.46.1, TransDecoder v.5.5.0, BLASTv.2.11.0, edgeRv.3.32.1.

Acknowledgements: The studies were funded by research grant No.19-76-00023 of the Russian Science Foundation and SA No. 0829-2019-0038 of the FSFIS "NBG-NSC" on the basis of the Unique Scientific Installation "FITOBIOGEN" FSFIS "NBG-NSC". 